\title{
Tips for Connecting Your Research with the Media
}

\author{
Kim Knott \\ Professor of Religious and Secular Studies \\ Lancaster University, UK \\ k.knott@lancaster.ac.uk \\ Solange Lefebvre \\ Chaire en gestion de la diversité culturelle et religieuse \\ Université de Montréal \\ solange.lefebvre@umontreal.ca
}

During the Religion and Diversity Project's 2015 annual team meeting, team members and local journalists came together for a panel on presenting research results to the media. Following the lively discussion, on the basis of our experience of researching and working with the media, we were asked to identify key points for communicating research in the press, on radio and television. Here are our top five tips for media engagement, contextualized by our experiences and professional backgrounds.

\section{Kim Knott, Lancaster University}

Engaging with the media is only an extension of the desire to communicate that most academics share, as lecturers and writers. It is the means and the audience that differ. My own media experiences have as often been 'behind the scenes' as in front of a microphone or on camera. The research contribution we can offer to those putting together programmes or stories is at least as important as our role as experts on breaking news.

Two occasions remain in my memory, one as an achievement: when I helped put together and then presented a $\mathrm{BBC}$ radio programme on nineteen sixties counter-culture, $\mathrm{Om}$ 1968. I learnt what a great medium radio is for research on religion.

I remember the other simply because it was entertaining. I was invited onto my local radio drive-time show to answer the question, "What is Gouranga?" The word could be seen painted on motorway bridges and on walls along railway tracks, but what did it mean? The show's researchers had gone out to ask passers-by their views; only one came close. It was fun to explain the mystery behind the word on air. Gouranga is another name for Krishna's 15th century Bengali disciple, Chaitanya. Hare Krishna devotees believe that Lord Krishna is manifested when his names are chanted, so to get people saying "Gouranga" is to invoke Krishna and his close disciples. This fulfils Chaitanya's plea, that the name of Krishna be spread to every town and village. Today's Krishna devotees are keeping this tradition alive in new ways. And we were doing the same on the radio that night.

Here are my five tips. 
1. Be proactive: provide your university or college press office with a list of things you feel confident to discuss with the media. Try to make these broad rather than narrow.

2. Get some media training, especially for television work. Even though you may not feel comfortable with role playing in a training scenario, it is really helpful for pointing out good and bad practice, what you should avoid and what works well.

3. If you are called by a journalist or media researcher, don't feel obliged to speak to them there and then. Find out what they want to know then say you'll call them back. Take 10 minutes to think about your response.

4. If you're invited to talk about your research or findings, work out in advance what your key points are (max 3$)$ and try to get them across. But if you're called for your expert opinion, try to be more flexible, if possible fitting your knowledge to the journalist's questions.

5. See if you can turn your research or expertise into a story or a succinct and compelling example. That works better than lots of numbers or data. It's about distinguishing the wood from the trees.

\section{Solange Lefebvre, Université de Montréal}

During my doctoral studies, I hesitated between journalism and an academic career. Even though I ended up choosing an academic career, I always enjoyed working with the media. Whether it was through radio or televised interviews, opinion pieces or conversations with journalists on current issues, I have engaged with the media dozens of times. One of my most memorable experiences was collaborating with the Canadian private broadcaster CTV during the 2013 conclave that elected Pope Francis. It must be noted that I was part of a large team covering the event in Rome that was led by the great journalist Lisa Laflamme. This was at the same time fascinating, intense and pleasant. The team's professionalism was remarkable. My experiences with journalists are, in most cases, always positive and significant. The five following tips aim to make the experiences of the experts in religion equally stimulating.

1. Be generous with your time. Academics often complain that journalists know little about religious issues. It is true. But the knowledge of religious matters is our profession. We must therefore collaborate with the media as good and patient pedagogues without seeking only visibility. It is important that we consider ourselves resource persons.

2. Take the opportunity to learn. Academic expertise is not always rooted in current events, more often it keeps a distance from them. Also, when contacted by a journalist, aren't we often tempted to say 'No' and argue that we do not understand the question thoroughly? Yet, the expertise implies that, unlike journalists, we must be able to quickly 
become aware of a matter and produce a sound and accurate analysis. This punctual analysis of facts can often be integrated in a course or in subsequent papers.

3. Every media has its own format and rules. Every medium has its own set of rules, and we have to master them well to contribute to the media sphere with relevance. The easiest form of media for experts to master is the opinion letter whose format is limited. Avoid doing what a colleague of mine once did. I received a note from him, complaining that his letter had been refused by the written press. His letter was 1500 words! I was very surprised that this retired colleague was not aware of the simple formatting rule. The statement must be clear and summarised. Moreover, it must be linked to current events that are making the news. It is useless to solicit a major written media in order to comment on an issue six months after its eruption. The train has left the station...

4. Respond quickly. With some exceptions, a journalist will be looking for a resource person that same day. If need be, she will contact several other individuals. It is then necessary not to hesitate and to respond quickly. But of course, we must avoid requests that are unpleasant, either because of the particular journalist, or because of the presence of another guest with whom we do not like to discuss.

5. Analyse the less positive experiences. A media intervention is fast and we may be disappointed with the journalist or with ourselves. We cannot exaggerate the significance of a failure in the media, we must bounce back and do better next time. After a few years, we can roughly anticipate all the moves and react to all types of situations. Just like politicians, we must learn sometimes to be put in a bad light in all humility.

There are many different opportunities for engaging your research with the media or even for becoming the 'go-to academic' on a particular subject. Even if journalists or media producers don't contact you, try blogging or tweeting. They offer practice in how to make your research accessible and succinct for a public audience. Short podcasts are another. You remain in control but you get the opportunity to summarise what you're an expert on in five minutes.

Working with the media, or with other stakeholders (practitioners, policy-makers, members of the public), is of mutual benefit. Media producers get content, of which they need a never-ending supply, and we get to represent and convey our research and expertise to a wider audience. For all the risks and anxieties associated with media engagement, it should be win-win! 